Original Contribution

\title{
COMPARATIVE STUDIES ON TWO TESTS FOR DETECTION OF AVIAN INFLUENZA A VIRUS AND NEWCASTLE DISEASE VIRUS IN CO-INFECTED GUINEA FOWL (NUMIDA MELEAGRIS)
}

\author{
Iv. Zarkov* \\ Department of Microbiology, Infections and Parasitic Diseases, Faculty of Veterinary Medicine, \\ Trakia University, Stara Zagora, Bulgaria
}

\begin{abstract}
Thirty four oropharyngeal swabs were collected from guinea fowl infected with a low-pathogenicity avian influenza A virus H6N2 (LPAIV H6N2) and vaccinated with a lentogenic NDV strain La Sota. All samples were examined in $\mathrm{HI}$ test after attempts for isolation of viruses in 9-day-old chick embryos (CE) and by means of AIV-NDV rapid Ag kit (RapiGEN, South Korea). The results demonstrated that the rapid test could be used for guinea fowl despite its lower sensitivity of $91.67 \%$ as compared to the $\mathrm{HI}$ test after isolation in CE. The test specificity was $100 \%$ indicating that it could distinguish both viruses in co-infections.
\end{abstract}

Key words: Avian influenza virus, Newcastle disease virus (NDV), AIV-NDV Rapid Ag kit, Virus isolation, $\mathrm{HI}$ test

\section{INTRODUCTION}

The differential diagnosis of some viral infections in birds e.g. caused by avian influenza virus (AIV), Newcastle disease virus (NDV), infection bronchitis virus, Marek disease virus, infectious bursal disease virus is difficult and often misleading by reason of comparable clinical signs, gross pathology findings and epidemiology (morbidity, mortality and lethality). These facts impede the proper tentative diagnostics. An additional obstacle is the presence of mixed infections in large avian populations with various etiological, immunological or vaccination history.

On the other hand, the early detection of infections with AIV and/or NDV is important to take adequate measures for confinement of the occurring disease and economic losses, as well as for implementation of efficient programmes of eradication and control.

To cope with some of these difficulties, rapid diagnostic tests have been developed, also suitable for in-field use. A problem related to

\footnotetext{
*Correspondence to: Ivan Zarkov, Department of Microbiology, Infections and Parasitic Diseases, Faculty of Veterinary Medicine, Trakia University, Stara Zagora, Bulgaria, E-mail: ivan_zarkov@abv.bg
}

their application is the insufficient knowledge on their diagnostic potential, period of test depending on the amount and serotype of the challenging virus. Each of developed tests has its advantages and flaws but comparative data on their performance in co-infections and avian species in which they could be used are not available $(1,2)$.

Several tests are available for diagnostics of avian influenza A virus, some of them are for detection of $\mathrm{H} 5$ and $\mathrm{H} 7$. The gold standard for comparison of test specificity and sensitivity is the isolation of the virus in chick embryos (CE). Test used frequently to determine the AIVls haemagglutinin type is the haemagglutinin inhibition $(\mathrm{HI})$ reaction $(3,4)$. The test takes several days.

Rapid tests of various manufacturers (QuickVue Influenza A + B; BinaxNow Influenza $\mathrm{A} \& \mathrm{~B}$; Directigen Flu $\mathrm{A}+\mathrm{B}$; Directigen EZ Flu A + B; Poctem Influenza A/B; Rapid Testa Flu II ) have been developed for detection of nucleoprotein (NP) or M1 protein. They are most appropriate in viral titres between $10^{3}$ and $10^{4} \mathrm{EID}_{50}$ and during the first 3-5 days of infection (5 - 7).

The substantial genetic diversity of NDV renders difficult its laboratory diagnostics. The isolation of the virus in CE (gold standard) is used for validation of other tests. After 
isolation $(85 \%$ of isolates are from the first passage), additional testing with other methods is necessary to differentiate isolates. A commonly used differentiation test is $\mathrm{HI}(8,9)$. Rapid tests for NDV detection based on immunochromatographic analysis are poorly developed and only few commercial products are marketed. It is known that these techniques could me misleading if the birds are vaccinated. Furthermore, they do not give any information about the pathogenicity and the genotype of the strain.

The aim of the present study was to compare the performance of a rapid test for detection of AIV and/or NDV and of HI test after isolation of the virus in CE in co-infected guinea fowl.

\section{MATERIAL AND METHODS}

\section{Viruses}

A low-pathogenicity avian influenza A virus H6N2 and vaccinal NDV strain La Sota were used.

\section{Samples}

Thirty four oropharyngeal swabs were collected from guinea fowl infected with a low-pathogenicity avian influenza A virus H6N2 (LPAIV H6N2) and vaccinated orally with a lentogenic NDV strain La Sota.

\section{Methods}

\section{A. Virus isolation (VI) and HI test for AIV H6 and NDV.}

Virus isolation and detection of haemagglutinin type as $\mathrm{H} 6$ of AIV and/or NDV with hyperimmune monospecific sera was done as described by Zarkov \& Valchev, 2017 (10).

Table 1. Comparative results of virus isolation with HI identification of NDV and rapid chromatographic test detection of NDV antigen in guinea fowl oropharyngeal samples

Isolation in 9-day-old chick embryos and $\mathrm{HI}$ identification of NDV

Rapid chromatographic test for detection of NDV

\begin{tabular}{|l|c|c|c|}
\hline & + & - & Total \\
\hline+ & 22 & 0 & 22 \\
\hline- & 2 & 10 & 12 \\
\hline Total & 24 & 10 & $\mathrm{n}=34$ \\
\hline
\end{tabular}

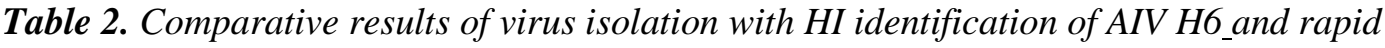
chromatographic test detection of AIV antigen in guinea fowl oropharyngeal samples

Isolation in 9-day-old chick embryos and $\mathrm{HI}$ identification of AIV H6

Rapid chromatographic test for detection of AIV
B. Test of AIV-NDV Rapid Ag kit

(RapiGEN, South Korea).

The test was performed according to the manufacturer's instructions. Results of the test were observed within 3-5 minutes, recorded by naked eye detection of single band for negative control, double band for AIV or NDV positive and triple band both for AIV and NDV

\section{Statistical analysis}

significance of methods was agreement of methods was carried out by the

\section{RESULTS} NDV test for viral antigens detection are presented in Table 1 and 2. Out of studied swab samples, $70.59 \%$ were $\mathrm{HI}$ positive after isolation of NDV and positive AIV H6 $35.29 \%$. The results from the rapid test were $64.71 \%$ positive for NDV and $32.35 \%$ positive Negative for NDV in the HI test were $29.41 \%$ of samples while this percentage in the rapid test was $35.29 \%$. In AIV $\mathrm{H6}$ testing, $\mathrm{HI}-$ negative samples were $64.71 \%$, vs $67.65 \%$ in the rapid test.

NDV-negative samples in the rapid test although positive in the HI test were 5.9\%. The respective percentage for AIV H6 was 2.94\%.

\begin{tabular}{|l|c|c|c|}
\hline & + & - & Total \\
\hline+ & 11 & 0 & 11 \\
\hline- & 1 & 22 & 23 \\
\hline Total & 12 & 22 & $\mathrm{n}=34$ \\
\hline
\end{tabular}


The results showed that the sensitivity of the rapid test for NDV compared to isolation in CE and $\mathrm{HI}$ identification of viruses was $91.67 \%$, with $100 \%$ specificity and agreement of both tests $94.12 \%$. Positive predictive value was $100 \%$, while negative predictive value: $83.34 \%$. The agreement between both tests was very high $(\mathbf{K}$ value -0.9$)$.

The data observed for AIV detection with isolation and subsequent identification with $\mathrm{HI}$ and the rapid AIV-NDV demonstrated that the sensitivity of the rapid test as compared to isolation with $\mathrm{HI}$ was $91.67 \%$, with $100 \%$ specificity and agreement of $97.06 \%$. Positive and negative predictive values were $100 \%$ and $95.65 \%$, respectively. The agreement between both tests for AIV detection was very high (K value -0.94$)$.

\section{DISCUSSION}

The AIV-NDV Rapid Ag kit is designed to test samples from chickens and ducks. Our experiments with guinea fowl samples showed that it could be successfully used with this species both for detection of AIV and NDV antigens. Our results support the data reported by Rahman et al., 2012 (2), that the test was very easy and rapid, less laborious, less time consuming and non expensive for the detection and differentiation of AIV and NDV.

All samples that were negative in the rapid AIV-NDV Ag test kit had low haemagglutination titres in the HI test: 1:8 and $1: 16$. This result was seen although in kit description, the threshold for detection of antigens was 0.125 HAU for AIV and 0.25 HAU за NDV.

According to Cattoli et al., 2004 (1), RyanPoirier et al., 1992 (12), Chambers et al., 1994 (13), Davison et al., 1998 (14) rapid tests were less sensitive for detection of AIV (79 \% - 86 $\%)$. Others (Chan et al., 2007) (5) established lower clinical sensitivity of samples from humans infected with avian influenza virus H5N1. Our previous studies with Directigen FLU A тест $(6,7)$ showed that it was less sensitive than the AIV-NDV Rapid Ag kit, presumably due to the fact that Directigen FLU A was developed for tests of influenza infection in humans while the AIV-NDV Rapid Ag kit was designed for use in birds. Furthermore, the present tests with AIV-NDV Rapid $\mathrm{Ag}$ kit and isolation with $\mathrm{HI}$ identification showed a very high agreement.

The evidenced $100 \%$ specificity of the AIVNDV Rapid Ag test kit showed that it could differentiate both viral species despite the few samples with different results.

\section{CONCLUSION}

The isolation of AIV and NDV in chick embryos and isolate identification in the HI test is accepted as gold standard for evaluation of the performance of other diagnostic tests. A disadvantage of VI and $\mathrm{HI}$ identification is the long time required for obtaining a result. Unlike it, the AIV-NDV Rapid Ag test kit provides a results within minutes, is easy to perform and reliable enough in guinea fowl coinfected with AIV and NDV.

\section{REFERENCES}

1. Cattoli,G., Drago, A., Maniero, S., Toffan, A., Bertoli, E., Fassina, S., Terregino, C., Robbi, C., Vicenzoni, G., Capua, I. Comparison of three rapid detection systems for type A influenza virus on tracheal swabs of experimentally and naturally infected birds. Avian Pathology, 33(4): 432-437, 2004.

2. Rahman, M. S., Rabbani, M. G., Uddin, M. J., Chakrabartty, A., Moon H. Prevalence of Avian Influenza and Newcastle Disease Viruses Using Rapid Antigen Detection Kit in Poultry in Some Areas of Bangladesh. Archives of Clinical Microbiology, 3, No.1:3 2012, doi:10.3823/248.

3. Capua, I. and Alexander, D. Avian influenza and Newcastle Disease. A field and laboratory manual, ISBN 978-88-4700825-0, Springer, Verlag Italia, 1-186, 2009.

4. OIE. Version adopted by the World Assembly of Delegates of the OIE in May 2015, OIE Terrestrial Manual, Chapter 2.3.4., Avian Influenza: 1-23, 2015.

5. Chan, K.H., Lam, S.Y., Puthavathana, P., Nguyen, T.D., Long, H., Pang, C.M., Chan,K.M., Cheung, C.Y., Seto, W., Peiris, J.S.M. Comparative analytical sensitivities of six rapid influenza $\mathrm{A}$ antigen detection test kits for detection of influenza A subtypes H1N1,H3N2 and H5N1. Journal of Clinical Virology, 38: 169 -171, 2007.

6. Zarkov, Iv. Use of commercial immunoassay for rapid detection of influenza A antigen in experimentally infected turkeys. Journal of Veterinary Record, 162: 126 - 127, 2008.

7. Zarkov, Iv. Comparison of the avian influenza virus detection by the method of viral re-isolation and by the Directigen ${ }^{\mathrm{TM}}$ Flu A test applied to cloacal and oropharyngeal samples from experimentally infected chickens and turkeys, Revue Medicine Veterinare, 159, 1: 49-53, 2008. 
8. Alexander, D. J. and Senne, D. A. Newcastle disease. In: Diseases of Poultry. $12^{\text {th }}$ edition. Edited by Saif Y.M., Glisson J.R., Mc Dougald L.R., Nolan, L.K., Swayne D. E. Ames, Iowa, USA: Blackwell Publishing; 75 - 100, 2008.

9. OIE. Newcastle disease. Version adopted by the World Assembly of Delegates of the OIE in May 2012. OIE Terrestrial Manual, Chapter 2.3.14:1-19, 2012.

10.Zarkov, I., Al. Valchev, Virus shedding in co-infections of low pathogenic avian influenza virus (H6N2) and lentogenic Newcastle disease virus (La Sota) in numida meleagris. Tracia Jurnal of sciences, 2: 113-120, 2017.

11.Courtney C.H. and Cornell, J.A. Evaluation of heartworm immunodiagnostic tests.
J.Am.Vet. Med. Assoc., 197, 6: 724-729, 1990.

12.Ryan-Poirier, K.A., Katz, J.M., Webster, R.G., Kawaoka, Y. Application of Directigen FLU-A for the detection of influenza $\mathrm{A}$ virus in human and nonhuman specimens. J. Clin. Microbiol.; 30:10721075, 1992.

13.Chambers, T.M., Shortridge, K.F., Li, P.H., Powell, D.G., Watkins, K.L. Rapid diagnosis of equine influenza by the Directigen FLU-A enzyme immunoassay. The Veterinary Record, 135: 275 -279, 1994.

14.Davison, S., Ziegler, A.F., Eckroade, R.J. Comparison of an antigen-capture enzyme immunoassay with virus isolation for avian influenza from field samples. Avian Diseases, 42: 791-795, 1998. 